University of Wollongong

Research Online

Faculty of Engineering and Information

Faculty of Engineering and Information

Sciences - Papers: Part A

Sciences

2012

Double space-time transmit diversity for very high data rate MB-OFDM UWB systems

Ngoc P. Le

University of Wollongong, pn1750@uowmail.edu.au

Le C. Tran

University of Wollongong, Ictran@uow.edu.au

Farzad Safaei

University of Wollongong, farzad@uow.edu.au

Follow this and additional works at: https://ro.uow.edu.au/eispapers

Part of the Engineering Commons, and the Science and Technology Studies Commons

Research Online is the open access institutional repository for the University of Wollongong. For further information contact the UOW Library: research-pubs@uow.edu.au 


\title{
Double space-time transmit diversity for very high data rate MB-OFDM UWB systems
}

\begin{abstract}
In this paper, we investigate a multiband-orthogonal frequency division multiplexing ultra-wideband (MBOFDM UWB) system with very high data rates (VHDR) of several gigabits per second (Gbps). We propose a DSTTD-based VHDR MB-OFDM UWB system by incorporating a double space-time transmit diversity (DSTTD) scheme into the conventional MB-OFDM UWB system. At the receiver, an efficient soft-demapper that takes into consideration the noise enhancement due to a zero-forcing equalization is developed. Numerical simulations of the proposed system at a very high data rate of 2.Gbps over the IEEE 802.15.3a channel models are implemented. The simulation results show that this system could achieve a very good performance in a low signal-to-noise ratio (SNR) region.
\end{abstract}

\section{Keywords}

data, double, space, time, ofdm, uwb, mb, transmit, diversity, very, systems, high, rate

Disciplines

Engineering | Science and Technology Studies

\section{Publication Details}

N. P. Le, L. C. Tran \& F. Safaei, "Double space-time transmit diversity for very high data rate MB-OFDM UWB systems," in ISCIT 2012: 12nd IEEE International Symposium on Communications and Information Technologies, 2012, pp. 1-5. 


\title{
Double Space-Time Transmit Diversity for Very High Data Rate MB-OFDM UWB Systems
}

\author{
Ngoc Phuc Le, Le Chung Tran, Farzad Safaei \\ School of Electrical, Computer and Telecommunications Engineering \\ The University of Wollongong \\ Northfields Avenue, NSW 2522, Australia \\ Emails: \{pnl750, lctran, farzad\}@uow.edu.au
}

\begin{abstract}
In this paper, we investigate a multiband-orthogonal frequency division multiplexing ultra-wideband (MB-OFDM UWB) system with very high data rates (VHDR) of several gigabits per second (Gbps). We propose a DSTTD-based VHDR MB-OFDM UWB system by incorporating a double space-time transmit diversity (DSTTD) scheme into the conventional MBOFDM UWB system. At the receiver, an efficient soft-demapper that takes into consideration the noise enhancement due to a zero-forcing equalization is developed. Numerical simulations of the proposed system at a very high data rate of 2 Gbps over the IEEE 802.15.3a channel models are implemented. The simulation results show that this system could achieve a very good performance in a low signal-to-noise ratio (SNR) region.
\end{abstract}

Keywords- D-STTD; MB-OFDM UWB; MDCM; Alamouti code; IEEE 802.15.3a channel models

\section{INTRODUCTION}

Ultra-wideband (UWB) communications has been considered as a promising transmission technique for short range, very high data rate applications [1]. Recently, Multiple Input Multiple Output (MIMO) schemes, including space time codes [2] and spatial multiplexing approaches [3], have been deployed in many wireless systems to significantly improve error performance and/or spectral efficiency.

The combination of MIMO and Multiband-OFDM UWB systems have been mentioned in the literature, such as [4], [5]. In [4], the authors proposed a space-time-frequency (STF) coded MB-OFDM UWB system under channels with a lognormal distribution. Meanwhile, in [5], the authors considered spatial multiplexing MIMO for MB-OFDM UWB systems. These works only investigated the systems with harddemapping and do not offer very high data rates. In [6], we proposed a STF coded VHDR MB-OFDM UWB system that could achieve a good performance at data rate of 1 Gbps by using the Alamouti STF code. In order to have MIMO MBOFDM UWB systems that could offer data speeds of up to several gigabits per second, besides LDPC codes and the MDCM modulation as mentioned in [7], the use of more than two transmit and/or receive antennas should be considered.
It is well-known that the double space-time transmit diversity (DSTTD) could provide a practical tradeoff between multiplexing gain and diversity gain [8]. This coding scheme is a hybrid combination of space time block codes and spatial multiplexing techniques. Besides the advantages of offering diversity gain and high data rates, the DSTTD can be used in OFDM-based systems over frequency selective fading channels. In fact, the DSTTD has been applied to many practical wireless systems, such as WLAN [9].

In this paper, we consider the application of the DSTTD in the VHDR MB-OFDM UWB system to boost data rates up to several Gbps. Specifically, we develop encoding and decoding procedures over space, time, and frequency dimensions at the transmitter and receiver, respectively. Based on the DSTTD scheme, the proposed DSTTD-based VHDR MB-OFDM UWB system could offer diversity gain and multiplexing gain simultaneously. It will be shown that this system could achieve a good error performance with low complexity.

The remainder of the paper is organized as follows. In Section II, we first describe the DSTTD scheme and then review the WiMedia's VHDR MB-OFDM specifications. In Section III, we propose the DSTTD-based VHDR MB-OFDM UWB system. Simulation results are provided in Section IV. Finally, Section V concludes the paper.

Notation: Throughout the paper, a bold letter denotes a vector or a matrix, whereas an italic letter denotes a variable. $(.)^{*},(.)^{\mathrm{T}},(.)^{-1}$, and $(\bullet)$ denote complex conjugation, transpose, inverse, and the Hadamard product, respectively. $\tilde{a}$ denotes an estimation of $a$. In addition, $\operatorname{Re}\{$.$\} and \operatorname{Im}\{$.$\} refer to the real$ and imaginary parts of a complex number, respectively.

\section{BACKGROUNDS}

\section{A. Double Space-Time Transmit Diversity}

Double space-time transmit diversity (DSTTD) [8] is a combination of spatial multiplexing and space-time block codes. The block diagram of a DSTTD system with four transmit antennas and two receive antennas is shown in Fig.1. 


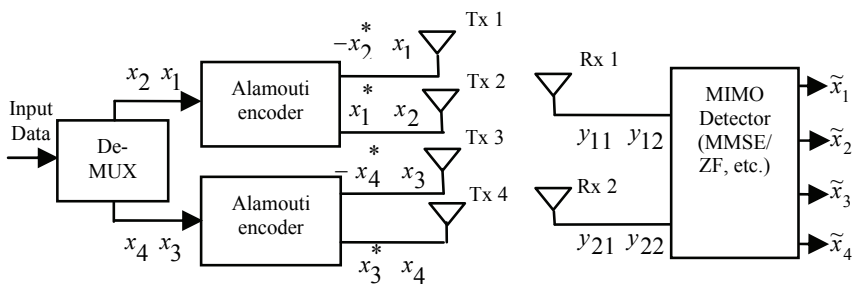

Fig. 1 Block diagram of a DSTTD system

At the transmitter, the input symbols are demultiplexed into two independent streams. Each stream is then encoded by an Alamouti encoder [2]. Assuming that the fading channel coefficients are constant for two symbol periods, the system model could be expressed in an equivalent form as [8]

$$
\mathbf{y}=\mathbf{H x}+\mathbf{n}
$$

where

$$
\begin{aligned}
\mathbf{x} & =\left[\begin{array}{llll}
x_{1} & x_{2} & x_{3} & x_{4}
\end{array}\right]^{T} \\
\mathbf{y} & =\left[\begin{array}{llll}
y_{11} & y_{12}^{*} & y_{21} & y_{22}^{*}
\end{array}\right]^{T} \\
\mathbf{n} & =\left[\begin{array}{llll}
n_{11} & n_{12}^{*} & n_{21} & n_{22}^{*}
\end{array}\right]^{T} \\
\mathbf{H} & =\left[\begin{array}{cccc}
h_{11} & h_{12} & h_{13} & h_{14} \\
h_{12}^{*} & -h_{11}^{*} & h_{14}^{*} & -h_{13}^{*} \\
h_{21} & h_{22} & h_{23} & h_{24} \\
h_{22}^{*} & -h_{21}^{*} & h_{24}^{*} & -h_{23}^{*}
\end{array}\right]
\end{aligned}
$$

Equation (1) shows that there is an equivalence between the transmission model of the DSTTD system and spatial multiplexing MIMO systems. Therefore, many detection techniques for MIMO system, such as zero-forcing (ZF) or minimum mean-squared error (MMSE), could be applied to detect signals at the receiver. In particular, when a ZF detector is used, the equalized signals are given as

$$
\widetilde{\mathbf{x}}=\mathbf{W y}=\mathbf{x}+\left(\mathbf{H}^{\prime} \mathbf{H}\right)^{-1} \mathbf{H}^{\prime} \mathbf{n}
$$

where $\mathbf{W}=\left(\mathbf{H}^{\prime} \mathbf{H}\right)^{-1} \mathbf{H}^{\prime}$ is a filter matrix for ZF. Although this linear detector suffers from an issue of noise enhancement, its complexity is very low. In particular, it is shown in [10] that this method does not require matrix inversion.

\section{B. Overview of WiMedia's VHDR MB-OFDM UWB PHY}

In the MB-OFDM approach [7], the entire UWB spectrum between 3.1-10.6 GHz is divided into 14 subbands, each has a bandwidth of $528 \mathrm{MHz}$. These subbands are grouped into 6 band groups as shown in Fig. 2.

The physical layer architecture of a VHDR MB-OFDM system is similar to that of a conventional OFDM one, except that the carrier frequency changes from one symbol to another. The block diagram of the transmitter is depicted in Fig. 3. Accordingly, information data are first scrambled, and then encoded by a LDPC encoder [11]. The encoded bit stream is mapped into a MDCM (Modified Dual Carrier Modulation) constellation [7]. The resulting data are fed into an IFFT block to generate OFDM symbols. The total number of subcarriers

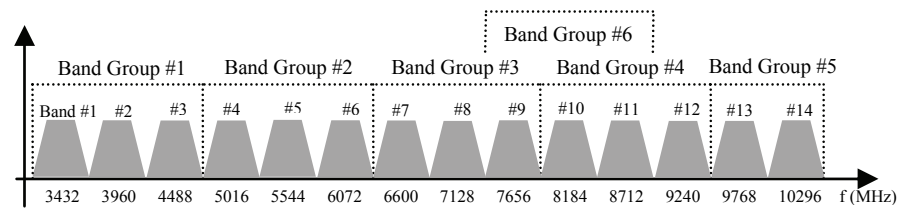

Fig. 2 MB-OFDM band group allocation

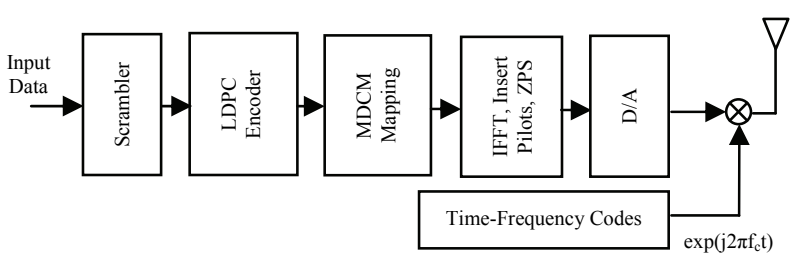

Fig. 3 Transmit architecture for a VHDR MB-OFDM system

in each OFDM symbol is 128 , of which, there are 100 data subcarriers, 12 pilot subcarriers, 10 guard subcarriers, and the rest are null subcarriers. At the receiver, the signals are processed in the reverse order.

With respect to some characteristics the VHDR MBOFDM system differs from the conventional OFDM system. First, a zero-padded suffix (ZPS) of length 37 is used to mitigate the effects of multipath as well as provide a guard interval for transceivers to switch from one subband to another. Second, the MB-OFDM system transmits symbols using different subbands specified by time-frequency codes (TFCs). These TFCs are used not only for providing frequency diversity but also for multiple access purpose. Third, wireless channels in a MB-OFDM UWB system follow a log-normal distribution, rather than the Rayleigh distribution.

\section{Table-based mapping for MDCM Scheme}

For the MDCM modulation, each group of eight bits is converted into two complex numbers, i.e. two OFDM subcarriers, $x(k)$ and $x(k+50), k \in[0,49]$. The mapping process is mathematically described in the WiMedia's specifications [7]. In [6], we proposed a table-based mapping approach that is useful for deriving soft-demapping expressions. To make this paper self-contained, we briefly summarize it as follows.

The table-based mapping for MDCM process is based on Table I. Denote a group of 8 bits needed to be mapped to be $b_{0} b_{1} b_{2} b_{3} b_{4} b_{5} b_{6} b_{7}$. The value of the bits $b_{0} b_{1} b_{4} b_{5}$ will determine the row from which the real parts of $x(k)$ and $x(k+50)$ should be selected. Meanwhile, the imaginary parts will be determined by the value of the bits $b_{2} b_{3} b_{6} b_{7}$. We now take a group of 8 bits (11001010) as an example. The value $b_{0} b_{l} b_{4} b_{5}=(1110)$ will be corresponding to the $12^{\text {th }}$ row. Therefore, we have $\operatorname{Re}\{x(k)\}=7$ and $\operatorname{Re}\{x(k+50)\}=-11$. 
TABLE I. A MAPPING TABLE FOR MDCM.

\begin{tabular}{|c|rrrrrrrrr|r|r|r|r|}
\hline No & $\mathrm{b}_{0} \mathrm{~b}_{1} \mathrm{~b}_{2} \mathrm{~b}_{3} \mathrm{~b}_{4} \mathrm{~b}_{5} \mathrm{~b}_{6} \mathrm{~b}_{7}$ & $\operatorname{Re}\left\{x_{k}\right\}$ & $\operatorname{Im}\left\{x_{k}\right\}$ & $\operatorname{Re}\left\{x_{k+50}\right\}$ & $\operatorname{Im}\left\{x_{k+50}\right\}$ \\
\hline 1 & 0 & 0 & 0 & 0 & 0 & 0 & 0 & 0 & -15 & -15 & 9 & 9 \\
2 & 0 & 0 & 0 & 0 & 0 & 1 & 0 & 1 & -13 & -13 & 1 & 1 \\
3 & 0 & 0 & 0 & 0 & 1 & 1 & 1 & 1 & -11 & -11 & -7 & -7 \\
4 & 0 & 0 & 0 & 0 & 1 & 0 & 1 & 0 & -9 & -9 & -15 & -15 \\
5 & 0 & 1 & 0 & 1 & 0 & 0 & 0 & 0 & -7 & -7 & 11 & 11 \\
6 & 0 & 1 & 0 & 1 & 0 & 1 & 0 & 1 & -5 & -5 & 3 & 3 \\
7 & 0 & 1 & 0 & 1 & 1 & 1 & 1 & 1 & -3 & -3 & -5 & -5 \\
8 & 0 & 1 & 0 & 1 & 1 & 0 & 1 & 0 & -1 & -1 & -13 & -13 \\
9 & 1 & 1 & 1 & 1 & 0 & 0 & 0 & 0 & 1 & 1 & 13 & 13 \\
10 & 1 & 1 & 1 & 1 & 0 & 1 & 0 & 1 & 3 & 3 & 5 & 5 \\
11 & 1 & 1 & 1 & 1 & 1 & 1 & 1 & 1 & 5 & 5 & -3 & -3 \\
12 & 1 & 1 & 1 & 1 & 1 & 0 & 1 & 0 & 7 & 7 & -11 & -11 \\
13 & 1 & 0 & 1 & 0 & 0 & 0 & 0 & 0 & 9 & 9 & 15 & 15 \\
14 & 1 & 0 & 1 & 0 & 0 & 1 & 0 & 1 & 11 & 11 & 7 & 7 \\
15 & 1 & 0 & 1 & 0 & 1 & 1 & 1 & 1 & 13 & 13 & -1 & -1 \\
16 & 1 & 0 & 1 & 0 & 1 & 0 & 1 & 0 & 15 & 15 & -9 & -9 \\
\hline
\end{tabular}

Similarly, for the bits $b_{2} b_{3} b_{6} b_{7}=(0010)$, by looking up to the $4^{\text {th }}$ row in the table, we obtain $\operatorname{Im}\{x(k)\}=-9$ and $\operatorname{Im}\{x(k+50)\}=-15$. Therefore, the values $x(k)$ and $x(k+50)$ are $(7-9 j)$ and $(-11-15 j)$, respectively.

We have some important observations from the above mapping. First, the values $\operatorname{Re}\{x(k)\}$ and $\operatorname{Re}\{x(k+50)\}$ depend only on the bits $b_{0}, b_{1}, b_{4}, b_{5}$, whereas the values $\operatorname{Im}\{x(k)\}$ and $\operatorname{Im}\{x(k+50)\}$ depend only on the bits $b_{2}, b_{3}, b_{6}, b_{7}$. Therefore, the computation of $\widetilde{b}_{i}, i=0,1,4,5$, depends only on $\operatorname{Re}\{\widetilde{x}(k)\}$ and $\operatorname{Re}\{\tilde{x}(k+50)\}$. Likewise, the computation of $\widetilde{b}_{i}, i=2,3,6,7$, depends on $\operatorname{Im}\{\widetilde{x}(k)\}$ and $\operatorname{Im}\{\tilde{x}(k+50)\}$. Second, the relation between the bits $b_{0}, b_{1}, b_{4}, b_{5}$ and $\operatorname{Re}\{x(k)\}$ is exactly the same as that between the bits $b_{2}, b_{3}, b_{6}, b_{7}$ and $\operatorname{Im}\{x(k)\}$. Also, the relation between the bits $b_{0}, b_{1}, b_{4}, b_{5}$ and $\operatorname{Re}\{x(k+50)\}$ is similar to that between the bits $b_{2}, b_{3}, b_{6}, b_{7}$ and $\operatorname{Im}\{x(k+50)\}$. Consequently, the steps required to obtain $\widetilde{b}_{i}, i=0,1,4,5$, from $\operatorname{Re}\{\tilde{x}(k)\}$ and $\operatorname{Re}\{\tilde{x}(k+50)\}$ is exactly the same as those needed to get $\tilde{b}_{i}, i=2,3,6,7$, from $\operatorname{Im}\{\tilde{x}(k)\}$ and $\operatorname{Im}\{\tilde{x}(k+50)\}$.

\section{DSTTD-BASED VHDR MB-OFDM UWB SYSTEM}

In this section, we propose the DSTTD-based VHDR MBOFDM UWB system. We consider a system equipped with $n_{T}=4$ transmit antennas and $n_{R}$ receive antennas $\left(n_{R} \geq 2\right)$. The block diagram of this system is depicted in Fig. 4. The operation of this system is described below.

At the transmitter, the data bits are encoded, and mapped onto MDCM constellation points. The resulting complex signal is then fed into the double space-time transmit diversity

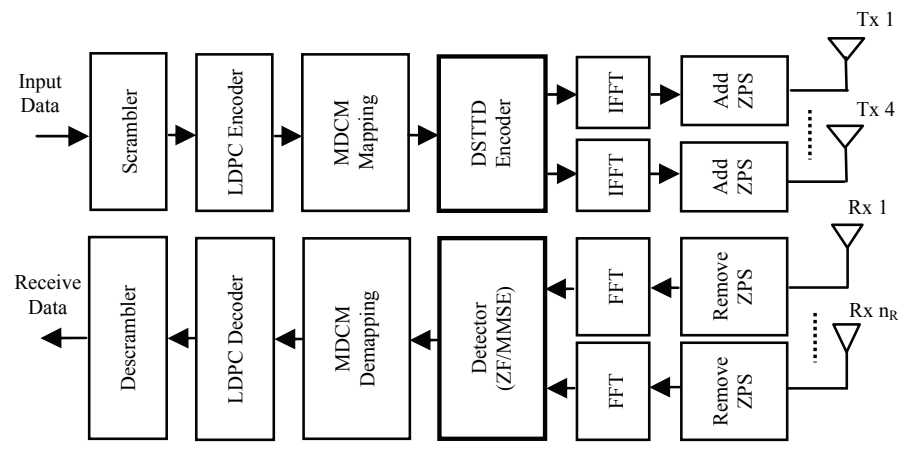

Fig. 4 Block diagram of a DSTTD-based VHDR MB-OFDM UWB system

encoder. The encoding process is similar to the one described in Section II.A. Denote $\mathbf{x}_{m}=\left[x_{m}(0), \ldots, x_{m}(K-1)\right], m=1,2,3,4$, to be the four blocks of $K=100$ complex numbers that the DSTTD encoder takes for each coding operation. In the first period, the encoder outputs $\mathbf{x}_{1}, \mathbf{x}_{2}, \mathbf{x}_{3}$, and $\mathbf{x}_{4}$ for transmission via the first, second, third, and fourth antenna, respectively. In the next period, the four corresponding output sequences are $-\mathbf{x}_{2}^{*}, \mathbf{x}_{1}^{*},-\mathbf{x}_{4}^{*}$, and $\mathbf{x}_{3}^{*}$. Therefore, the STF transmission matrix could be expressed as

$$
\mathbf{X}=\left[\begin{array}{rrrr}
\mathbf{x}_{1} & \mathbf{x}_{2} & \mathbf{x}_{3} & \mathbf{x}_{4} \\
-\mathbf{x}_{2}^{*} & \mathbf{x}_{1}^{*} & -\mathbf{x}_{4}^{*} & \mathbf{x}_{3}^{*}
\end{array}\right]^{\mathrm{T}}
$$

The output sequences from the encoder are then fed into 128 point IFFT blocks. Each IFFT output sequence is added with a zero-padded suffix (ZPS) to form a MB-OFDM symbol before being transmitted via its corresponding transmit antenna.

At the receiver, the received signal at each antenna is fed into the FFT block after the ZPS is removed. Denote $\mathbf{y}_{n}^{1}$ and $\mathbf{y}_{n}^{2}$ to be the resulting signals from the $n^{\text {th }}$ receive antenna in the first and second period, respectively. These signals could be expressed as [4]

$$
\begin{aligned}
& \mathbf{y}_{n}^{1}=\mathbf{H}_{n, 1} \bullet \mathbf{x}_{1}+\mathbf{H}_{n, 2} \bullet \mathbf{x}_{2}+\mathbf{H}_{n, 3} \bullet \mathbf{x}_{3}+\mathbf{H}_{n, 4} \bullet \mathbf{x}_{4}+\mathbf{N}_{n}^{1} \\
& \mathbf{y}_{n}^{2}=-\mathbf{H}_{n, 1} \bullet \mathbf{x}_{2}^{*}+\mathbf{H}_{n, 2} \bullet \mathbf{x}_{1}^{*}-\mathbf{H}_{n, 3} \bullet \mathbf{x}_{4}^{*}+\mathbf{H}_{n, 4} \bullet \mathbf{x}_{3}^{*}+\mathbf{N}_{n}^{2}
\end{aligned}
$$

where $\mathbf{y}_{n}^{p}=\left[y_{n}^{p}(0), \ldots, y_{n}^{p}(K-1)\right], p=1,2 ; n=1,2, \ldots, n_{R}$, are the received signals, $\mathbf{H}_{n, m}=\left[H_{n, m}(0), H_{n, m}(1) \ldots, H_{n, m}(K-1)\right]$ is the channel frequency response of the path between the $m^{\text {th }}$ transmit antenna and the $n^{\text {th }}$ receive antenna that remains constant during the two consecutive periods of transmission, and $\mathbf{N}_{n}^{p}, p=1,2$, are independent noise samples modelled as Gaussian random variables with variance $\sigma^{2}$ per dimension.

At the DSTTD decoder, assuming perfect channel state information (CSI) is available, the equalized signal corresponding to each subcarrier for each transmit data stream is obtained by using the method that requires no matrix inversion mentioned in the Section II.A. The four estimated 
sequences, denoted as $\widetilde{\mathbf{x}}_{m}, m=1,2,3,4$, are then taken by the MDCM demapper to produce the soft-value for each bit.

Define the log-likelihood ratio (LLR) of the $i^{\text {th }}, \mathrm{i}=0, \ldots, 7$ bit associated with the pair of symbols $x_{m}(k), x_{m}(k+50)$ as $\operatorname{LLR}\left(b_{i, m}\right)=\log \frac{\operatorname{Pr}\left[b_{i, m}=1 \mid H_{n, m}(k), H_{n, m}(k+50), \widetilde{x}_{m}(k), \widetilde{x}_{m}(k+50)\right]}{\operatorname{Pr}\left[b_{i, m}=0 \mid H_{n, m}(k), H_{n, m}(k+50), \widetilde{x}_{m}(k), \widetilde{x}_{m}(k+50)\right]}$

Using the first observation mentioned in Section II.C, for estimating bits $\tilde{b}_{i, m}, i=0,1,4,5$, we could express the LLR values as in (10) (see at the bottom), where $S_{i, m}^{(0)}$ and $S_{i, m}^{(1)}$ are the sets of the real parts of constellation symbols whose $i^{\text {th }}$ bit is 0 and 1 , respectively. As the two subcarriers, $k^{\text {th }}$ and $(k+50)^{\text {th }}$, are separated by at least $200 \mathrm{MHz}$ in UWB systems [7], it is possible to assume that $\tilde{x}_{m}(k)$ and $\tilde{x}_{m}(k+50)$ are independent. Define $\Sigma_{m}^{2}(k)$ to be the variance of the noise after ZF filtering that is associated with $\tilde{x}_{m}(k)$. For a ZF detector, assuming that all the transmitted symbols are equally likely, by applying the property of a joint Gaussian probability density function (pdf) and Bayes' rule, we have (11), where the joint Gaussian pdf $f\{$.$\} could be expressed by (12), and$ (13) [12]. Using the log-map approximation, we finally have (14). Based on the second observation in Section II.C, the LLR expression for bits $\tilde{b}_{i, m}, i=2,3,6,7$ are obtained by replacing $\operatorname{Re}\left\{\widetilde{x}_{m}(k)\right\}$ and $\operatorname{Re}\left\{\widetilde{x}_{m}(k+50)\right\}$ in (14) with $\operatorname{Im}\left\{\tilde{x}_{m}(k)\right\}$ and $\operatorname{Im}\left\{\tilde{x}_{m}(k+50)\right\}$, respectively. Also, $S_{i, m}^{(0)}$ and $S_{i, m}^{(1)}$ are now the corresponding sets of the imaginary parts of symbols. Moreover, these LLR values could be further normalized as done in [13]. It should be noted that, in the final
TABLE II. SimUlation Parameters.

\begin{tabular}{|l|l|}
\hline \multicolumn{1}{|c|}{ Parameter } & \multicolumn{1}{|c|}{ Value } \\
\hline Data rate & $2048 \mathrm{Mbps}$ \\
FFT size & 128 \\
Number of information data subcarriers & 100 \\
Modulation scheme & MDCM \\
LDPC code (code rate of 4/5) & Table 6.32 in [7] \\
Channel models & $\mathrm{CM} 1, \mathrm{CM}, \mathrm{CM}, \mathrm{CM} 4$ \\
Number of channel realizations & 100 \\
\hline
\end{tabular}

LLR expressions in (14), we reflect the CSI with respect to the post-filtering noise variance. Therefore, the system performance is expected to be improved with this soft demapper [14].

Finally, the LDPC decoder takes these soft-values for softdecoding in order to retrieve the transmitted information.

\section{Simulation Results}

In this section, simulation results are provided to illustrate the performance of the proposed system. The simulation parameters are listed in Table II. These parameters are chosen based on the WiMedia's specifications for the data rate of 1024 Mbps. Hence, the data rate in the proposed system employing the DSTTD scheme is 2048 Mbps. In addition, the LDPC encoding algorithm developed in [11] which could achieve linear running time is adopted in our simulation. We measure the system performance in terms of packet error rate (PER) over the IEEE 802.15.3a channel models defined in [15]. There are four channel models, namely CM1, CM2, CM3, and CM4, corresponding to different scenarios. The CM1 channel model is based on a measurement of a Line of Sight (LOS) scenario where the distance between the transmitter and the receiver is up to $4 \mathrm{~m}$. The others are CM2 $(0-4 \mathrm{~m}$, Non Line of SightNLOS), CM3 (4-10m, NLOS), CM4 (4-10m, NLOS, rms delay spread of $25 \mathrm{~ns})$. Moreover, the multipath gains are modeled as independent log-normally distributed random variables in these models. We assume that perfect CSI is available at the receiver.

$$
\begin{aligned}
& \operatorname{LLR}\left(b_{i, m}\right)=\log \left(\frac{\sum_{\left(\alpha_{1}, \alpha_{2}\right) \in S_{i, m}^{(1)}} \operatorname{Pr}\left[\operatorname{Re}\left\{x_{m}(k)\right\}=\alpha_{1}, \operatorname{Re}\left\{x_{m}(k+50)\right\}=\alpha_{2} \mid H_{n, m}(k), H_{n, m}(k+50), \operatorname{Re}\left\{\tilde{x}_{m}(k)\right\}, \operatorname{Re}\left\{\tilde{x}_{m}(k+50)\right\}\right]}{\sum_{\left(\beta_{1}, \beta_{2}\right) \in S_{i, m}^{(0)}} \operatorname{Pr}\left[\operatorname{Re}\left\{x_{m}(k)\right\}=\beta_{1}, \operatorname{Re}\left\{x_{m}(k+50)\right\}=\beta_{2} \mid H_{n, m}(k), H_{n, m}(k+50), \operatorname{Re}\left\{\tilde{x}_{m}(k)\right\}, \operatorname{Re}\left\{\tilde{x}_{m}(k+50)\right\}\right]}\right) \\
& \operatorname{LLR}\left(b_{i, m}\right)=\log \left(\frac{\sum_{\left(\alpha_{1}, \alpha_{2}\right) \in S_{i, m}^{(1)}} f_{\alpha}\left\{\operatorname{Re}\left\{\tilde{x}_{m}(k)\right\}, \operatorname{Re}\left\{\tilde{x}_{m}(k+50)\right\}\left|H_{n, m}(k), H_{n, m}(k+50), \operatorname{Re}\left\{x_{m}(k)\right\}=\alpha_{1}, \operatorname{Re}\left\{x_{m}(k+50)\right\}=\alpha_{2}\right|\right\}}{\sum_{\left(\beta_{1}, \beta_{2}\right) \in S_{i, m}^{(0)}} f_{\beta}\left\{\operatorname{Re}\left\{\tilde{x}_{m}(k)\right\}, \operatorname{Re}\left\{\tilde{x}_{m}(k+50)\right\}\left|H_{n, m}(k), H_{n, m}(k+50), \operatorname{Re}\left\{x_{m}(k)\right\}=\beta_{1}, \operatorname{Re}\left\{x_{m}(k+50)\right\}=\beta_{2}\right|\right\}}\right) \\
& f_{\alpha}\{.\}=\frac{1}{2 \pi \Sigma_{m}(k) \Sigma_{m}(k+50)} \exp \left(-\frac{\left[\operatorname{Re}\left\{\tilde{x}_{m}(k)\right\}-\alpha_{1}\right]^{2}}{2 \Sigma_{m}^{2}(k)}-\frac{\left[\operatorname{Re}\left\{\tilde{x}_{m}(k+50)\right\}-\alpha_{2}\right]^{2}}{2 \Sigma_{m}^{2}(k+50)}\right) \\
& f_{\beta}\{.\}=\frac{1}{2 \pi \Sigma_{m}(k) \Sigma_{m}(k+50)} \exp \left(-\frac{\left[\operatorname{Re}\left\{\tilde{x}_{m}(k)\right\}-\beta_{1}\right]^{2}}{2 \Sigma_{m}^{2}(k)}-\frac{\left[\operatorname{Re}\left\{\tilde{x}_{m}(k+50)\right\}-\beta_{2}\right]^{2}}{2 \Sigma_{m}^{2}(k+50)}\right) \\
& \operatorname{LLR}\left(b_{i, m}\right)=\left\{\begin{array}{c}
-\min _{\left(\alpha_{1}, \alpha_{2}\right) \in S_{i, m}^{(1)}}\left(\frac{\left[\operatorname{Re}\left\{\tilde{x}_{m}(k)\right\}-\alpha_{1}\right]^{2}}{2 \Sigma_{m}^{2}(k)}+\frac{\left[\operatorname{Re}\left\{\tilde{x}_{m}(k+50)\right\}-\alpha_{2}\right]^{2}}{2 \Sigma_{m}^{2}(k+50)}\right)+ \\
\min _{\left(\beta_{1}, \beta_{2}\right) \in S_{i, m}^{(0)}}\left(\frac{\left[\operatorname{Re}\left\{\tilde{x}_{m}(k)\right\}-\beta_{1}\right]^{2}}{2 \Sigma_{m}^{2}(k)}+\frac{\left[\operatorname{Re}\left\{\tilde{x}_{m}(k+50)\right\}-\beta_{2}\right]^{2}}{2 \Sigma_{m}^{2}(k+50)}\right)
\end{array}\right\}, i=0,1,4,5
\end{aligned}
$$


Fig. 5 shows the PER performance of the proposed system with different numbers of receive antennas. It is evident that the proposed system can achieve a target PER of $10^{-2}$ in a low SNR region. Moreover, there is a significant error performance improvement when the number of receive antennas increases. For example, a gain of about $4.5 \mathrm{~dB}$ is attained over the CM1 channel model in a system equipped with three receive antennas, compared to the case of using two receive antennas. This is due to the fact that the DSTTD scheme could offer an additional diversity gain when the number of receive antennas is larger than two [8].

Fig. 6 compares the performance of the proposed system with that of the spatial multiplexing (SM) based system at the same data rate of 2 Gbps. In this comparison, both systems have a $4 \mathrm{x} 4 \mathrm{MIMO}$ configuration. The $\mathrm{ZF}$ detector is used in the proposed system, whereas a MMSE detector is used in the SM system. Also, a 4-QAM modulation scheme is adopted in the SM system. Hence, all systems have an equal spectral efficiency of about $6 \mathrm{bits} / \mathrm{s} / \mathrm{Hz}$. It is clear that the proposed system outperforms the SM counterpart under all channel models. This advantage could be explained from the diversity gain perspective. Specifically, when the ZF or MMSE detector is used in a MIMO configuration with equal numbers of transmit and receive antennas, the SM system only has a diversity order of one. Meanwhile, the proposed system using the DSTTD scheme enjoys a higher diversity order [8]. We also observe a high error floor occurring in the CM4 channel. This is a result of using the simple ZF detection in the scenario where the channel impulse response of the CM4 channel is much longer than the zero-padded suffix.

\section{CONCLUSIONS}

A MB-OFDM UWB system that could offer very high data rates of up to $2 \mathrm{Gbps}$ has been proposed. This system is achieved by incorporating the efficient DSTTD scheme into the standard MB-OFDM UWB system. It is shown that this system could achieve a good PER performance at low SNR values with low complexity. We have considered the simple ZF detector for the DSTTD scheme in this paper. The use of other detection techniques might provide an improved error performance at the expense of extra computational cost.

\section{REFERENCES}

[1] L. Yang and G. B. Giannakis, "Ultra-wideband communications: an idea whose time has come," IEEE Signal Processing Magazine, vol. 21, no. 6, pp. 26-54, Nov 2004.

[2] S. M. Alamouti, "A simple transmit diversity technique for wireless communications," IEEE Journal on Selected Areas in Communications, vol. 16, no. 8, pp. 1451- 1458, Oct. 1998.

[3] G. D. Golden, C. J. Foschini, R. A. Valenzuela, and P. W. Wolniansky, "Detection algorithm and initial laboratory results using V-BLAST space-time communication architecture," IEEE Electronics Letters, vol. 35, no. 1, pp. 14-16, Jan. 1999.

[4] L. C. Tran and A. Mertins, "Space-time frequency code implementation in MB-OFDM UWB communications: design criteria and performance," IEEE Trans. Wireless Commun., vol. 8, no. 2, pp. 701-713, Feb. 2009.

[5] J. Adeane, W. Q. Malik, and I. J. Wassell, "Error performance of ultrawideband spatial multiplexing systems," IET Microwave, Antenna and Propagation, vol. 3, no. 3, pp. 363-371, April 2009.

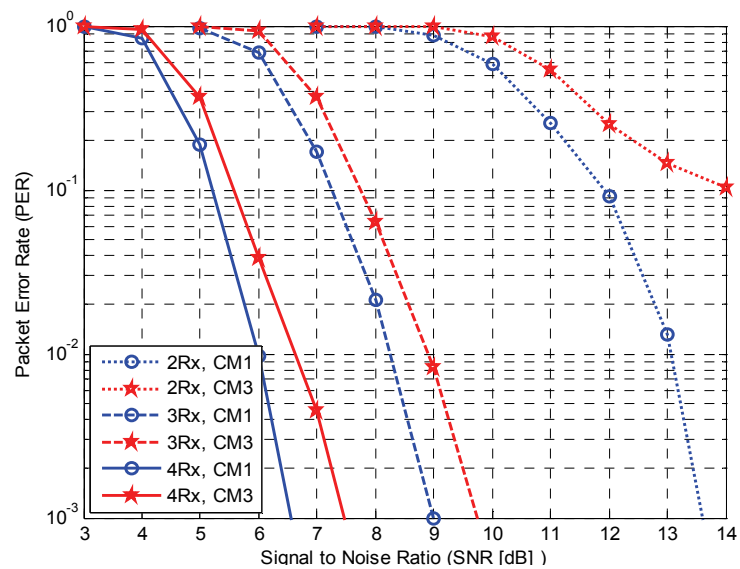

Fig. 5 Performance of the DSTTD-based VHDR MB-OFDM

UWB system with different numbers of receive antennas

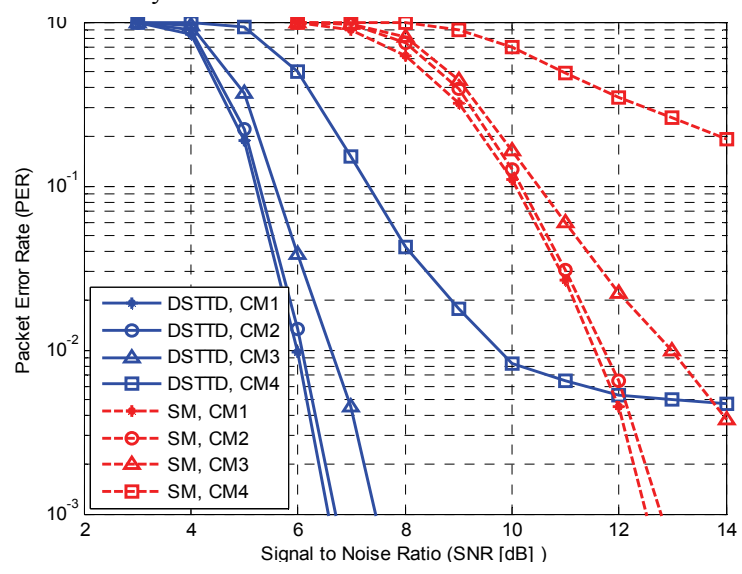

Fig. 6 Performance comparison of the DSTTD-based VHDR MB-OFDM UWB system with the SM system (4x4 MIMO)

[6] N. P. Le, L.C. Tran, and F. Safaei, "Very high data rate MB-OFDM UWB systems with transmit diversity techniques," IEEE International Symposium on Communications and Information Technologies, ISCIT 2012, Gold Coast, Australia, 2012. Accepted.

[7] A. Batra et al., "Multiband OFDM physical layer specification," WiMedia Alliance, Release 1.5, August 2009.

[8] E. N. Onggosanusi, A. G. Dabak, and T. M. Schmidl, "High rate spacetime block coded scheme: Performance and improvement in correlated fading channels," in Proc. IEEE Wireless Communications and Networking Conference, WCNC, vol. 1, pp. 194-199, March 2002.

[9] IEEE P802.11n/D1.0, Part 11: Wireless LAN Medium Access Control (MAC) and Physical Layer (PHY) specifications: Enhancements for Higher Throughput, March 2006.

[10] J. Ham, S. Shim, K. Kim, and C. Lee, "A simplified adaptive modulation scheme for D-STTD systems with linear receivers," IEEE Communications Letter, vol. 9, no. 12, pp. 1049-1051, Dec. 2005.

[11] T. J. Richardson and R. Urbanke, "Efficient encoding of low-density parity-check codes," IEEE Transactions on Information Theory, vol. 47, no. 2, pp. 638- 656, Feb. 2001.

[12] S. L. Miller and D. G. Childers, Probability and random processes: With applications to signal processing and communications, Elsevier Academic Press, 2004.

[13] F. Tosato and P. Bisaglia, "Simplified soft-output demapper for binary interleaved COFDM with application to HIPERLAN/2," in Proc. IEEE Int. Conf. Communications, ICC 2002, vol. 2, pp. 664-668, April 2002.

[14] C. Yoon, J. Son, and S. Lee, "A novel ZF detection scheme for double SFBC based OFDM system in frequency selective fading channel," in Proc. IEEE Int. Conf. on Comm., ICC 2007, pp. 2557-2561, June 2007.

[15] J. Foerster et al., "Channel modeling sub-committee report final," IEEE P802.15-02/490r1-SG3a, Feb. 2003. 